\title{
A novel COL1A2 C-propeptide cleavage site mutation causing high bone mass osteogenesis imperfecta with a regional distribution pattern
}

\author{
T. Rolvien ${ }^{1,2}$ • U. Kornak ${ }^{3,4,5}$ - J. Stürznickel ${ }^{1}$ - T. Schinke ${ }^{1}$ - M. Amling ${ }^{1}$ (D) \\ S. Mundlos ${ }^{3,4,5} \cdot$ R. Oheim ${ }^{1}$
}

Received: 19 July 2017 / Accepted: 7 September 2017 / Published online: 15 September 2017

(C) International Osteoporosis Foundation and National Osteoporosis Foundation 2017

\begin{abstract}
Osteogenesis imperfecta (OI) is typically characterized by low bone mass and increased bone fragility caused by heterozygous mutations in the type I procollagen genes (COL1A1/COL1A2). We report two cases of a 56-year-old woman and her 80-year-old mother who suffered from multiple vertebral and non-vertebral fractures with onset in early childhood. A full osteologic assessment including dual-energy X-ray absorptiometry (DXA), high-resolution peripheral quantitative computed tomography (HR-pQCT), and serum analyses pointed to a high bone mineral density (BMD) in the hip (DXA Z-score + 3.7 and + 3.9) but low to normal bone mass in the spine and preserved bone microstructure in the distal tibia. Serum markers of bone formation and bone resorption were elevated. Using whole exome sequencing, we identified a novel mutation in the COL1A2 gene causing a p. (Asp1120Gly) substitution at the protein level and affecting the type I procollagen C-propeptide cleavage site. In line with previously reported cases, our data independently prove the existence of an unusual phenotype of high bone mass OI
\end{abstract}

M. Amling

amling@uke.de

1 Department of Osteology and Biomechanics, University Medical Center Hamburg-Eppendorf, Lottestr. 59, 22529 Hamburg, Germany

2 Department of Orthopaedic Surgery, University Medical Center Hamburg-Eppendorf, Martinistraße 52, 20246 Hamburg, Germany

3 Institute of Medical Genetics and Human Genetics, Charité-Universitätsmedizin Berlin, 13353 Berlin, Germany

4 Berlin-Brandenburg School for Regenerative Therapies, Charité-Universitätsmedizin Berlin, Berlin, Germany

5 FG Development and Disease, Max Planck Institute for Molecular Genetics, Berlin, Germany caused by a mutation in the procollagen C-propeptide cleavage with a clinically persistent phenotype through adulthood.

\section{Introduction}

Osteogenesis imperfecta (OI) is a well described genetic skeletal disorder defined by low bone mass and increased bone fragility. Heterozygous mutations in two genes encoding collagen 1 (COL1A1/COL1A2) are present in most of the affected patients [1]. Specific COL1A1 [2-5] and COL1A2 [4, 6, 7] mutations affecting the $\mathrm{C}$-propeptide cleavage can be associated with high bone mass (HBM) and this may radiologically mimic autosomal dominant osteopetrosis type 2 .

\section{Patients and methods}

Here, we report a 56-year-old woman (II.1) and her 80-yearold mother (I.1), who experienced multiple vertebral and peripheral fractures with onset in early childhood and persistent fractures through adulthood (Fig. 1a, b). Osteogenesis imperfecta was suspected due to the clinical course but had not been genetically confirmed.

In order to further analyze the bone status, the areal bone mineral density (aBMD) was evaluated using dual-energy Xray absorptiometry (DXA, Lunar iDXA, GE Healthcare; Madison, WI, USA). DXA quality assurance was achieved following the institutional standard operating procedures (SOPs) and by daily calibration scans with a special phantom according to the manufacturer's recommendations. Furthermore, serum and urinary bone turnover markers were assessed. For bone microstructure analysis, a high-resolution peripheral quantitative computed tomography (HR-pQCT; XtremeCT, Scanco Medical, Switzerland) was performed at 
A

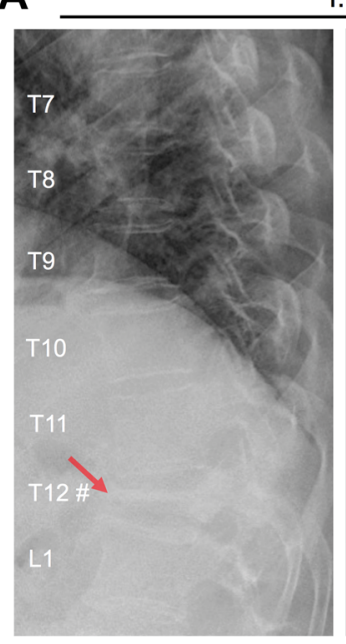

1.1

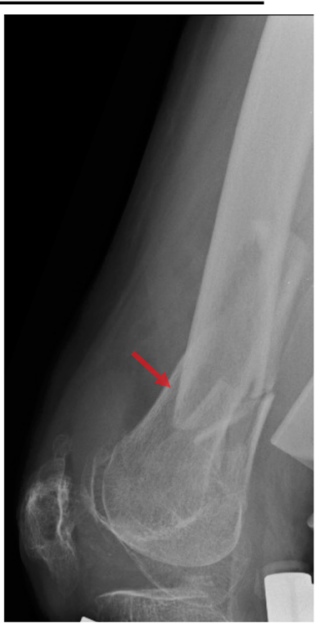

B

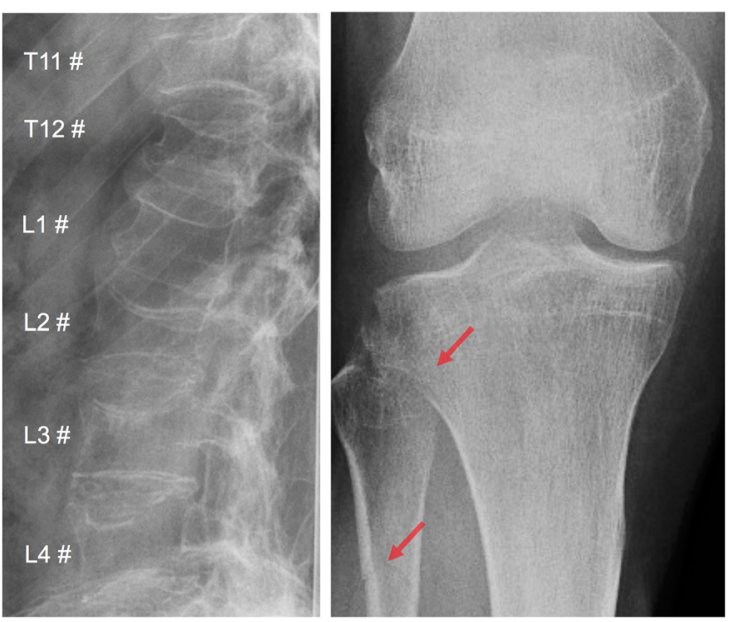

C

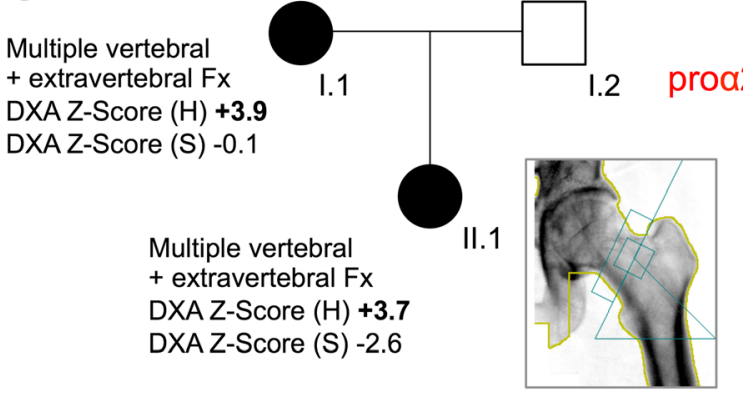

D

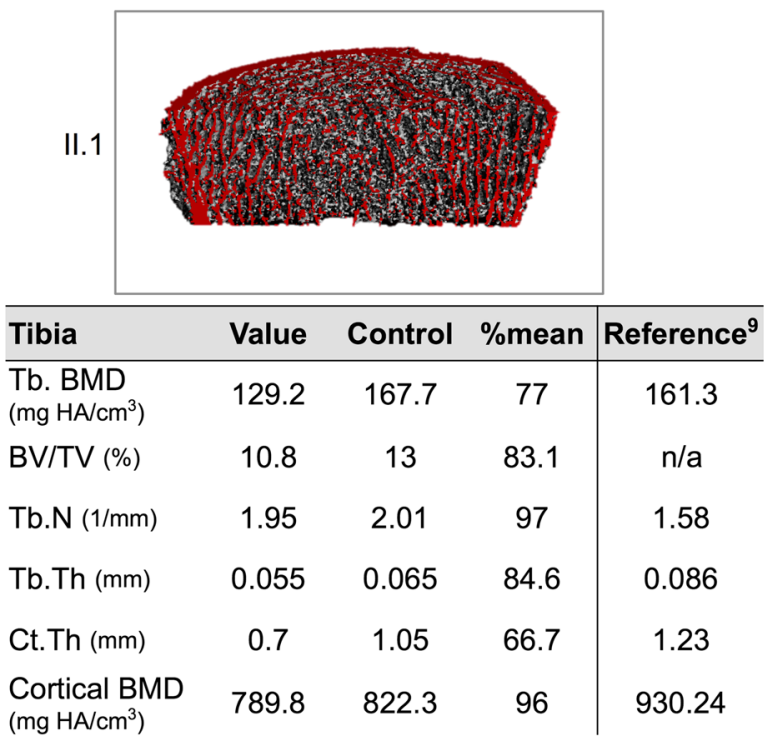

Fig. 1 Osteologic assessment in two patients (mother and daughter) with high bone mass OI. a Radiographs showing a vertebral compression fracture of T12 (left, red arrow), as well as a distal femur fracture in the affected mother (right, red arrow, I.1). b In the daughter (II.1), > 50 fractures had occurred in total including multiple vertebral fractures (left) and a fracture of the tibia plateau and proximal fibula (red arrows). c Pedigree including DXA Z-scores and schematic drawing indicating the dominant COL1A2 cleavage site mutation c.3359A $>\mathrm{G}$ (p.Asp1120Gly). H: femur/total hip; S: lumbar spine. d Bone

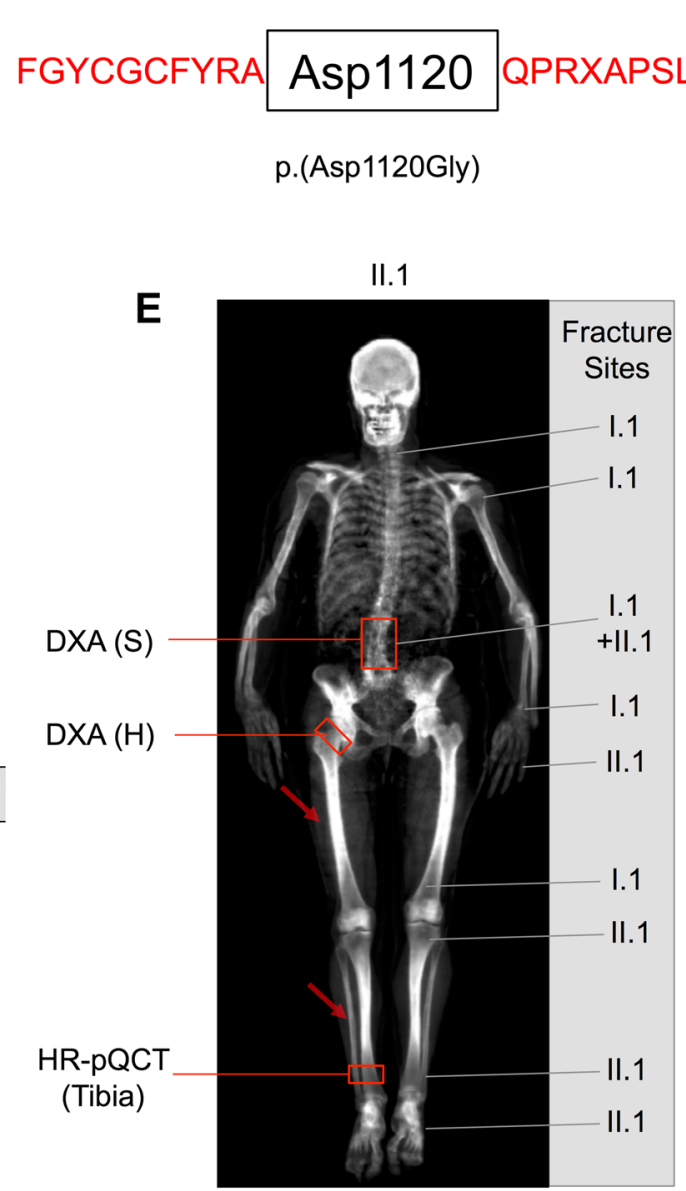

microstructure analysis using HR-pQCT in the tibia of case II.1 pointed to slightly decreased trabecular and cortical parameters compared to ageand sex-matched controls from our database as well as compared to reference data from the literature [9]. Tb: trabecular; BV/TV: bone volume/per tissue volume; Th: thickness; $\mathrm{Ct}$ : cortical; BMD: bone mineral density. e Whole body DXA indicating the high bone mass pattern that was most likely reserved to the hip, femur, and tibia diaphysis (red arrows). Red boxes indicate the measurement sites for hip and lumbar spine DXA, as well as HR-pQCT in the distal tibia 
the distal tibia in a standardized procedure using the in vivo protocol as previously described by our group [8]. HR-pQCT results were compared to a healthy age- and sex-matched controls from our database that underwent the HR-pQCT scan for screening purposes as well as to reference values from the literature (50th centile for 50-60 women) [9].

In the first part of the mutational analysis, all known genes leading to skeletal dysplasias with high bone mass including SOST, TCIRG1, TGFB1, OSTM1, SNX10, TNFSF11, TNFRSF11A, ANKH, LEMD3, FAM20C, LRP5, and CTSK were tested by next-generation sequencing (NGS) using a custom designed bone mass gene panel (Sure Select, Agilent). After no variants were detected in these genes, whole exome sequencing was carried out by enrichment of all coding genes. The sequencing was done on a HiSeq sequencing machine (Illumina, San Diego, CA, USA). Data were analyzed by the software tools GeneTalk and PhenIX [10]. The pathogenicity of the prioritized variants was judged using MutationTaster [11].

\section{Results}

The initial osteologic assessment by dual-energy X-ray absorptiometry (DXA) revealed a high bone mass in the hip (DXA Z-score total hip I.1 + 3.9; II.1 + 3.7), but normal to low BMD in the spine (Fig. 1c). Since analysis of genes causing high bone mass disorders was negative, exome sequencing was performed revealing a novel mutation in the COL1A2 gene (c.3359A $>$ G; NM_000089). The resulting p. (Asp1120Gly) substitution at the protein level resides at the type I procollagen C-propeptide cleavage site (Fig. 1c).

In contrast to the increased bone mineral density (BMD) in total hip DXA, high-resolution peripheral quantitative computed tomography (HR-pQCT) at the tibia of patient II.1 indicated a normal to low volumetric bone density and bone microstructure compared to a healthy age- and sex-matched controls from our database and reference values from the literature [9] (Fig. 1d). Whole body DXA scan essentially pointed out that the high bone mass was predominantly restricted to the hips, femur, and tibia diaphysis (Fig. 1e, red arrows), while the scanning volume from HR-pQCT as well as the lumbar spine DXA most likely covered a region with normal to low bone mass (Fig. 1e, red boxes).

Bone turnover serum analyses showed a high bone turnover state with adequate levels of bone formation markers (i.e., osteocalcin and bone-specific alkaline phosphatase) and elevated markers of bone resorption in the mother and daughter (Table 1).

Both patients had a relatively short stature (I.1 $151 \mathrm{~cm}$; II.1 $156 \mathrm{~cm}$ ). No blue sclerae, dentinogenesis imperfecta, or hearing loss were observed. Prior to their initial presentation at our clinic, they had received oral bisphosphonate medication
Table 1 Laboratory results including serum bone turnover markers

\begin{tabular}{llll}
\hline Parameter & I.1 & II.2 & Reference range \\
\hline $\mathrm{Ca}(\mathrm{mmol} / \mathrm{l})$ & 2.23 & 2.29 & $2.13-2.63$ \\
$25-\mathrm{OH}-\mathrm{D}_{3}(\mu \mathrm{g} / \mathrm{l})$ & $28.3(-)$ & 43.6 & $>30$ \\
$\mathrm{PTH}(\mathrm{ng} / \mathrm{l})$ & 28.0 & $87.2(+)$ & $17-84$ \\
$\mathrm{Oc}(\mu \mathrm{g} / \mathrm{l})$ & 14.2 & 20.3 & $5.4-59.1$ \\
$\mathrm{BAP}(\mu \mathrm{g} / \mathrm{l})$ & 20.3 & $27.0(+)$ & $5.2-24.4$ \\
$\mathrm{DPD}(\mathrm{nmol} / \mathrm{mmol})$ & $12(+)$ & $8(+)$ & $3-7$ \\
\hline
\end{tabular}

$\mathrm{Ca}$ Calcium, 25-OH- $\mathrm{D}_{3}$ 25-Hydroxyvitamin D, $\mathrm{PTH}$ parathyroid hormone, $O c$ osteocalcin $(\mathrm{Oc}), B A P$ bone-specific alkaline phosphatase, $D P D$ deoxypyridinoline cross-links in the urine

(alendronate) for years without relevant changes of bone mineral density measured by DXA and without any effect on fracture rate. Furthermore, patient I.1 developed osteonecrosis of the jaw (ONJ) under oral bisphosphonate treatment, which was therefore discontinued.

\section{Discussion}

The detected mutation p. (Asp1120Gly) has not been reported before, but the previously detected p. (Asp1120Ala) [7] and p. (Asp1120Asn) [6] mutations associated with high bone mass OI affect the same codon within the cleavage site of the alpha 2 chain of type 1 procollagen. In line with previously reported cases [2-7], our data independently prove the existence of an unusual phenotype of high BMD OI caused by inhibition of procollagen C-propeptide cleavage. Similarly, biallelic mutations in $B M P 1$, encoding the procollagen C-proteinase, have been found to lead to a high bone mass OI phenotype $[12,13]$. While the previously published high bone mass OI phenotypes have been seen in children with high bone mass and fractures, our results indicate a persistence of high bone mass through late adulthood. In the younger patients, [4] sclerotic vertebral bands are seen, which are absent in our two older patients. This indicates that this phenotypic aspect may depend on growth and may not be a reliable indicator for the diagnosis.

The high bone mass phenotype has been linked to increased matrix mineralization in biopsy studies using quantitative backscattered electron imaging (qBEI) and Fouriertransform infrared spectroscopy (FTIR) [4]. In this study, the elevated BMD Z-score in the hip was neither mirrored by high bone mass in lumbar spine DXA nor in HR-pQCT of the distal tibia, which has essentially not been yet performed in patients with high bone mass OI. This might be due to regional distribution pattern of increased bone mass depending on the skeletal site (i.e., diaphyseal, cortical not metaphyseal, cancellous). In fact, when examining the fracture sites of both patients, there were no fractures at the high bone mass sites, but occurred rather in the bone with presumable normal or low 
bone mass (i.e., atlas, proximal humerus, thoracic and lumbar spines, distal radius, proximal and distal tibia and fibula, calcaneus, distal femur, Fig. 1e). As the skeleton presents a strong heterogeneity in general [14], the site-dependent changes in bone density and microarchitecture in the presence of COL1 C-propeptide cleavage site mutations have to be further elucidated, i.e., by analyzing biopsies from common fracture sites.

In fact, a COL1A1 C-propeptide mutation was detected in a patient with a phenotype similar to gnathodiaphyseal dysplasia (GDD) including generalized sclerosis of the skull [5]. We have previously outlined the distinctive phenotype of GDD caused by ANO5 mutation that similarly involves high bone mass with predominant occurrence in the long bones [15]. There are no known interaction effects between ANO5 and type I collagen; however, bone turnover markers have been found to be elevated in GDD [15] as well as in this and other studies on OI patients [16]. Therefore, the existence of a complex disease mechanism causing impairments of bone mineralization or osteoclastic resorption is possible.

In conclusion, we demonstrated a novel COL1A2 Cpropeptide cleavage site mutation in a 56-year-old patient and her 80-year-old mother that expands the spectrum of high bone mass OI. In light of our and previous findings, both the effectiveness of a bone-specific therapy (i.e., bisphosphonate) and the mechanism leading to high bone mass at different skeletal sites, i.e., by inhibition of C-propeptide cleavage, have to be investigated further.

Funding information This project has received funding from the European Community's Seventh Framework Programme under grant agreement no. 602300 (SYBIL) and the German Federal Ministry of Education and Research (BMBF) within the project "Detection and Individualized Management of Early Onset Osteoporosis (DIMEOS).”

\section{Compliance with ethical standards}

Conflicts of interest None.

\section{References}

1. Forlino A, Marini JC (2016) Osteogenesis imperfecta. Lancet 387: 1657-1671

2. Pollitt R, McMahon R, Nunn J, Bamford R, Afifi A, Bishop N, Dalton A (2006) Mutation analysis of COL1A1 and COL1A2 in patients diagnosed with osteogenesis imperfecta type I-IV. Hum Mutat 27:716

3. Cundy T, King A, Byers PH (2008) A novel disorder of type I collagen characterised by high bone mass, a mineralization defect and tendon calcification. Calcif Tissue Int 82:S1
4. Lindahl K, Barnes AM, Fratzl-Zelman N, Whyte MP, Hefferan TE, Makareeva E, Brusel M, Yaszemski MJ, Rubin CJ, Kindmark A, Roschger P, Klaushofer K, McAlister WH, Mumm S, Leikin S, Kessler E, Boskey AL, Ljunggren O, Marini JC (2011) COL1 Cpropeptide cleavage site mutations cause high bone mass osteogenesis imperfecta. Hum Mutat 32:598-609

5. McInerney-Leo AM, Duncan EL, Leo PJ, Gardiner B, Bradbury LA, Harris JE, Clark GR, Brown MA, Zankl A (2015) COL1A1 Cpropeptide cleavage site mutation causes high bone mass, bone fragility and jaw lesions: a new cause of gnathodiaphyseal dysplasia? Clin Genet 88:49-55

6. Nishimura G, Nakajima M, Takikawa K, Haga N, Ikegawa S (2016) Distinctive skeletal phenotype in high bone mass osteogenesis imperfecta due to a COL1A2 cleavage site mutation. Am J Med Genet A 170:2212-2214

7. $\mathrm{Li} \mathrm{C}$ (2009) Increased bone mineral density and body mass index in families of osteogenesis imperfecta: a new type of OI? American Society of Human Genetics (ASHG), Honolulu annual meeting

8. Milovanovic P, Adamu U, Simon MJ, Rolvien T, Djuric M, Amling M, Busse B (2015) Age- and sex-specific bone structure patterns portend bone fragility in radii and tibiae in relation to osteodensitometry: a high-resolution peripheral quantitative computed tomography study in 385 individuals. J Gerontol A Biol Sci Med Sci 70(10):1269-1275

9. Burt LA, Liang Z, Sajobi TT, Hanley DA, Boyd SK (2016) Sexand site-specific normative data curves for HR-pQCT. J Bone Miner Res 31(11):2041-2047

10. Zemojtel T, Kohler S, Mackenroth L, Jager M, Hecht J, Krawitz P, Graul-Neumann L, Doelken S, Ehmke N, Spielmann M, Oien NC, Schweiger MR, Kruger U, Frommer G, Fischer B, Kornak U, Flottmann R, Ardeshirdavani A, Moreau Y, Lewis SE, Haendel M, Smedley D, Horn D, Mundlos S, Robinson PN (2014) Effective diagnosis of genetic disease by computational phenotype analysis of the disease-associated genome. Sci Transl Med 6: 252ra123

11. Schwarz JM, Rodelsperger C, Schuelke M, Seelow D (2010) MutationTaster evaluates disease-causing potential of sequence alterations. Nat Methods 7:575-576

12. Asharani PV, Keupp K, Semler O, Wang W, Li Y, Thiele H, Yigit G, Pohl E, Becker J, Frommolt P, Sonntag C, Altmuller J, Zimmermann K, Greenspan DS, Akarsu NA, Netzer C, Schonau E, Wirth R, Hammerschmidt M, Nurnberg P, Wollnik B, Carney TJ (2012) Attenuated BMP1 function compromises osteogenesis, leading to bone fragility in humans and zebrafish. Am J Hum Genet 90:661-674

13. Hoyer-Kuhn H, Semler O, Schoenau E, Roschger P, Klaushofer K, Rauch F (2013) Hyperosteoidosis and hypermineralization in the same bone: bone tissue analyses in a boy with a homozygous BMP1 mutation. Calcif Tissue Int 93:565-570

14. Amling M, Herden S, Posl M, Hahn M, Ritzel H, Delling G (1996) Heterogeneity of the skeleton: comparison of the trabecular microarchitecture of the spine, the iliac crest, the femur, and the calcaneus. J Bone Miner Res 11:36-45

15. Rolvien T, Koehne T, Kornak U, Lehmann W, Amling M, Schinke T, Oheim R (2017) A novel ANO5 mutation causing gnathodiaphyseal dysplasia with high bone turnover osteosclerosis. J Bone Miner Res 32:277-284

16. Braga V, Gatti D, Rossini M, Colapietro F, Battaglia E, Viapiana O, Adami S (2004) Bone turnover markers in patients with osteogenesis imperfecta. Bone 34:1013-1016 